\title{
Sox1 Is Required for the Specification of a Novel p2-Derived Interneuron Subtype in the Mouse Ventral Spinal Cord
}

\author{
Helen Panayi, ${ }^{1}$ Elena Panayiotou, ${ }^{1,2}$ Michael Orford, ${ }^{1}$ Nicolas Genethliou, ${ }^{1,2}$ Richard Mean, ${ }^{1}$ George Lapathitis, ${ }^{1}$ \\ Shengguo Li, ${ }^{3}$ Mengqing Xiang, ${ }^{3}$ Nicoletta Kessaris, ${ }^{4}$ William D. Richardson, ${ }^{4}$ and Stavros Malas ${ }^{1,2}$ \\ ${ }^{1}$ The Cyprus Institute of Neurology and Genetics, 2370 Nicosia, Cyprus, ${ }^{2}$ Department of Biological Sciences, University of Cyprus, 1678 Nicosia, Cyprus, \\ ${ }^{3}$ Center for Advanced Biotechnology and Medicine and Department of Pediatrics, University of Medicine and Dentistry of New Jersey, Robert Wood \\ Johnson Medical School, Piscataway, New Jersey 08854, and ${ }^{4}$ Wolfson Institute for Biomedical Research and Research Department of Cell and \\ Developmental Biology, University College London, London WC1E 6BT, United Kingdom
}

During mouse development, the ventral spinal cord becomes organized into five progenitor domains that express different combinations of transcription factors and generate different subsets of neurons and glia. One of these domains, known as the $\mathrm{p} 2$ domain, generates two subtypes of interneurons, V2a and V2b. Here we have used genetic fate mapping and loss-of-function analysis to show that the transcription factor Sox 1 is expressed in, and is required for, a third type of $\mathrm{p} 2$-derived interneuron, which we named V2c. These are close relatives of V2b interneurons, and, in the absence of Sox1, they switch to the V2b fate. In addition, we show that late-born V2a and V2b interneurons are heterogeneous, and subsets of these cells express the transcription factor Pax6. Our data demonstrate that interneuron diversification in the $\mathrm{p} 2$ domain is more complex than previously thought and directly implicate Sox 1 in this process.

\section{Introduction}

The ventral spinal cord (vSC) in mice is organized into five progenitor domains (ventral-p3-pMN-p2-p1-p0-dorsal), specified through the graded activity of sonic hedgehog released from the notochord and floor plate (Ericson et al., 1997a). Progenitor cells in each domain express different sets of homeodomain and basic helix-loop-helix transcription factors, which are primarily responsible for specifying the neuronal and glial lineages characteristic of that domain (Briscoe et al., 2000; Muhr et al., 2001; Hochstim et al., 2008). For example, the pMN domain generates different types of motor neurons (MNs), whereas the $\mathrm{p} 2$ domain generates at least two different types of interneurons (INs), named V2a and V2b. Neuronal diversity in the pMN domain is achieved through the action of extrinsic and intrinsic factors that influence the fates of postmitotic progenitors (Dalla Torre di Sanguinetto et al., 2008). Similarly, neuronal subtype specification in the $\mathrm{p} 2$ domain is determined in postmitotic progenitors through the action of Notch/Delta signaling in both mice (Del Barrio et al., 2007; Peng et al., 2007) and zebrafish (Batista et al., 2008; Kimura et al., 2008). Thus, postmitotic Notch ${ }^{\text {-high }}$ progenitors give rise to $\mathrm{V} 2 \mathrm{~b}$ INs, whereas Notch ${ }^{- \text {low }}$ progenitors acquire a V2a fate. Accordingly, attenuation of Notch signaling leads to overproduction of V2a INs at the expense of V2b INs (Yang et al., 2006; Del Barrio et al., 2007; Peng et al., 2007; Batista et al., 2008).

Received May 11, 2010; accepted July 10, 2010.

This work was supported by grants from the Research Promotion Foundation of Cyprus and the European Union. N.K. and W.D.R. are supported by the Medical Research Council (United Kingdom), The Wellcome Trust, and the European Union. We thank Frank Grosveld for the anti-GATA3 antibodies.

Correspondence should be addressed to Stavros Malas, The Cyprus Institute of Neurology and Genetics, Airport Avenue, №.6, Agios Dometios 2370, 1683 Nicosia, Cyprus. E-mail: smalas@cing.ac.cy.

DOI:10.1523/JNEUROSCI.2402-10.2010

Copyright $\odot 2010$ the authors $\quad 0270-6474 / 10 / 3012274-07 \$ 15.00 / 0$
Downstream of Notch, the action of the Lim-only protein (Lmo4) either favors or inhibits transcriptional complexes in subsets of postmitotic progenitors and consolidates the V2a and V2b IN fates (Joshi et al., 2009).

Sox 1 is a transcription factor belonging to Group B of the Sox gene family. It is expressed by most neural progenitors in the embryonic spinal cord (Pevny et al., 1998; Wood and Episkopou, 1999; Genethliou et al., 2009) and forebrain in which it is required for correct differentiation of postmitotic GABAergic neurons that contribute to the ventral striatum (Malas et al., 2003; Ekonomou et al., 2005).

In this study, we investigated the expression and function of Sox1 in a new group of INs that are generated in the vSC. We show that these cells derive from the $\mathrm{p} 2$ domain but are distinct from V2a and V2b INs. We named these cells V2c INs and show that they are lineally related to V2b INs. In the absence of Sox 1 , V2c INs become reprogrammed toward the V2b cell fate, suggesting that SOX1 is necessary for regulating the V2b versus V2c fate choice. Finally, we show that the transcription factor Pax6 is expressed in subsets of late-born V2a and V2b INs and possibly in a fourth type of $\mathrm{p} 2$-derived INs. These data reveal that the $\mathrm{p} 2$ domain generates more IN subtypes than originally appreciated and that Sox1 has an essential role in this diversification.

\section{Materials and Methods}

Transgenic mice. We used the following established mouse lines: Sox ${ }^{\mathrm{KO}}$ (Aubert et al., 2003), SOX1 ${ }^{\beta-\text { geo/+ }}$ (Malas et al., 2003), Pax6 (Sey allele) (Hill et al., 1991), Rosa26 ${ }^{\text {stopYFP }}$ (Srinivas et al., 2001), and Foxn $4^{\mathrm{KO}}$ (Li et al., 2004). We generated two additional lines, Foxn $4^{\text {-iCre }}$ and GATA3 ${ }^{\text {-eGFP }}$, using bacterial artificial chromosome (BAC) recombination as described previously (Zhang et al., 2000). The BACs used to generate Foxn $4^{-i C r e}$ and GATA $3^{-\mathrm{eGFP}}$ mouse lines were 150 and $180 \mathrm{~kb}$ long, respectively. To generate the BACs, we first made a purpose-built cassette containing 
either enhanced Green Fluorescent Protein (eGFP) or codon-improved Cre recombinase (iCre) coding sequences cloned upstream of a prokaryotic promoter (EM7) driving the kanamycin resistance gene, resulting in an iCre-FRT-EM7-Kan ${ }^{\mathrm{R}}$-FRT or eGFP-FRT-EM7-Kan ${ }^{\mathrm{R}}$-FRT cassette. These were then amplified under high-fidelity PCR conditions using long oligonucleotides with homology overhangs that corresponded to the region where recombination was desired. Recombination strategies were designed such that the coding sequences of the first exon of each gene were deleted ( $87 \mathrm{bp}$ for Foxn 4 and $286 \mathrm{bp}$ for Gata3). The sequences flanking the insertions were as follows: Foxn4, 5'atccctaaggaa-iCre-tacaggtgag-3'; Gata3, 5' agccgaggac-eGFP-cgtggaccca3' . After verification for correct recombination, the EM7-KanR was removed using Flipmediated excision in bacteria, so that only the eGFP or iCre gene was retained in the BAC. The molecular integrity of the final BACs was assessed by DNA fingerprinting using at least three restriction enzymes. Three founder lines of Foxn $4^{-i C r e}$ were established, which gave similar patterns of recombination with the Rosa26 $6^{\text {stopYFP }}$ reporter; the line that gave the most robust recombination was selected for additional study. Four Gata3 ${ }^{-e G F P}$ founder lines were generated that expressed eGFP in an identical manner to Gata3. One of these lines was selected for additional study. The Nkx2.2-CreERT2 BAC transgenic line will be reported later (R. Taveira-Marques, N.K., and W.D.R., unpublished observations). All animal procedures were performed in accordance with a license issued by the Chief of Veterinary Services of the Republic of Cyprus, according to National Law. Genotyping was done by PCR analysis. Pax6 mutant (Sey/ Sey) embryos were identified morphologically by the absence of eyes and by the shape of the telencephalon.

Immunohistochemistry. Immunohistochemistry was performed using embryos fixed in MEMFA [0.1 м 3-( $N$-morpholino)-propanesulfonic acid, $2 \mathrm{~mm} \mathrm{EGTA,} 1 \mathrm{~mm} \mathrm{MgSO}_{4}$, and 3.7\% formaldehyde] for a period of 15-35 min, depending on the age of the embryo and the antibodies used. This was followed by extensive washing in $0.1 \mathrm{M}$ PBS and cryoprotection in $15 \%(\mathrm{w} / \mathrm{v})$ sucrose. Sections $(10-12 \mu \mathrm{m})$ were cut on a cryostat. Antibody detection was performed using immunofluorescence according to standard procedures. The following primary antibodies were used: antiGFP (Invitrogen), anti-Nkx2.2, anti-Nkx6.1, anti-Evx1, anti-Isl1/2, and anti-PAX6 (Developmental Studies Hybridoma Bank, University of Iowa, Iowa City, IA), anti-Pax6 and anti-neuronal-specific nuclear protein (NeuN) (Millipore Bioscience Research Reagents), anti-Olig2, antiSox1, and anti-Chx10 (Santa Cruz Biotechnology), anti-Gata3 (gift from Frank Grosveld, Erasmus Medical Centre, Department of Cell Biology, Rotterdam, The Netherlands), anti-Foxn4 (Li et al., 2004), anti- $\beta$ galactosidase (Cappel, ICN Pharmaceuticals), and anti-Soxl (made in guinea pigs against the peptide AGGRHPHAHPAHPHPHНPHAHPHNPQP). Images were captured on a TCSL confocal microscope (Leica).

In situ hybridization. Embryos were fixed overnight in $4 \%(\mathrm{w} / \mathrm{v})$ paraformaldehyde in $0.1 \mathrm{M}$ phosphate buffer $(\mathrm{PB})$, cryoprotected overnight in $30 \%(\mathrm{w} / \mathrm{v})$ sucrose in $\mathrm{PB}$, and sectioned on a cryostat $(14 \mu \mathrm{m})$. In situ hybridization was performed using digoxigenin-labeled RNA probes. The following probes were used: Foxn 4 and iCre. The probes were generated by PCR amplification of either mouse genomic DNA or cloned cDNA with sequence-specific primers, ligated into pGEM-T Easy (Promega) and amplified by directional PCR to generate the desired template for antisense probe synthesis. Images were captured using an Olympus microscope (model SZX12) and digital camera (Olympus model DP70).

Cell counting and statistical analysis. Serial sections from equivalently staged embryos at forelimb level were used for cell counting. For each stage and genotype, three embryos were used to obtain 10 sections for counting, totaling 30 sections. The quantitative results were analyzed by two-tailed distribution, homoscedastic Student's $t$ test. The graphed results are shown as means \pm SEM.

\section{Results}

\section{SOX1 is expressed in a subset of interneurons in the ventral} spinal cord

In the developing spinal cord at embryonic day 9.5 (E9.5), Sox 1 is expressed only in ventricular zone (VZ) progenitors and not differentiated cells (Pevny et al., 1998; Genethliou et
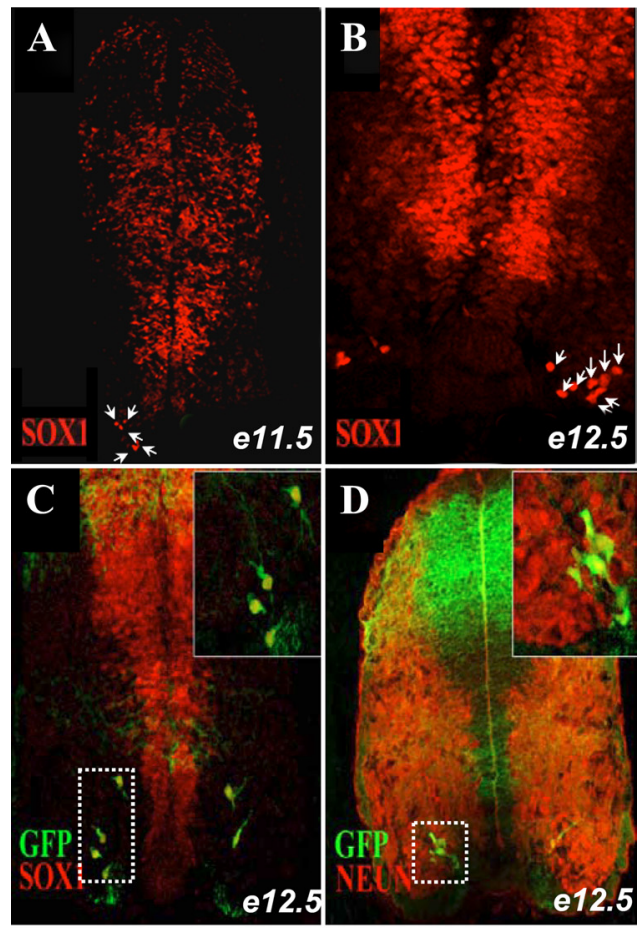

E

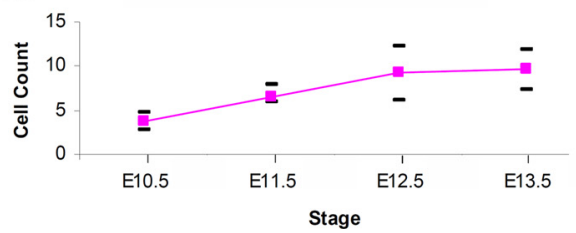

Figure 1. SOX1 is expressed in a group of ventral interneurons. Spinal cord sections from wild-type $(\boldsymbol{A}, \boldsymbol{B})$ and Sox $1^{\mathrm{GFP} /+}(\boldsymbol{C}, \boldsymbol{D})$ embryos at the stages denoted were immunostained with the antibodies indicated. Arrows in $\boldsymbol{A}$ and $\boldsymbol{B}$ indicate the Sox ${ }^{+}$neurons. Insets in $\boldsymbol{C}$ and $\boldsymbol{D}$ show a higher magnification of the areas enclosed in dotted boxes in the respective panels. $\boldsymbol{E}$ shows a quantification of the mean \pm SD number of Sox $1^{+}$neurons observed in 30 sections at forelimb level at the indicated stages. Note that the number of Sox $1^{+}$neurons becomes stable between $\mathrm{E} 12.5$ and $\mathrm{E} 13.5$.

al., 2009). However, from late E10.5, in addition to the VZ expression, we detected Sox 1 expression in a small group of differentiated cells migrating close to the p3 domain (Fig. $1 A, B$ and data not shown). These cells, monitored by GFP expression in Sox 1 GFP/+ embryos (carrying a GFP "knock-in" allele) (Fig. 1C), expressed the pan-neuronal marker NeuN (Fig. 1D) and had short axonal processes that extended within the spinal cord. The production of these cells peaked at approximately E12.5, and their average number ranged from 7 to 16 cells per $12 \mu \mathrm{m}$ section (Fig. $1 E$ ).

We tested a battery of markers, including Nkx2.2 expressed by V3 neurons and their progenitors (Briscoe et al., 1999), Isl1 and Isl2 expressed by motor neurons (Thaler et al., 2002), Evx1 expressed in ventrally migrating V0 INs (Moran-Rivard et al., 2001), Chx10 expressed by V2a INs, and Gata3 expressed by V2b INs (Karunaratne et al., 2002; Lundfald et al., 2007). None of these markers colocalized with GFP in Sox 1 GFP/+ embryos (supplemental Figs. $1 A-E, 3 C, E$, available at www.jneurosci.org as supplemental material). These data suggest that Sox1 is not only a marker for $\mathrm{VZ}$ progenitors but is also expressed by a small group of ventral neurons, most likely INs, that do not express any markers of known IN subtypes. 
Sox1-positive neurons originate from the $\mathbf{p} 2$ domain

Based on their final resting position, it seemed likely that the Sox1-positive $\left(\right.$ Sox $\left.1^{+}\right)$INs originated in either the $\mathrm{p} 3$ or p2 domains, because the intervening pMN domain is believed to generate only motor neurons and glia (Masahira et al., 2006). To distinguish these possibilities, we examined $N k x 2.2^{-C r e E R T 2}$ transgenic mice and generated Foxn $4^{-i C r e}$ mice by pronuclear injection of a BAC transgene. $\mathrm{Nkx} 2.2$ is expressed by p3 progenitors (Briscoe et al., 2000), whereas Foxn4 is expressed by all committed p2-derived neuronal progenitors (Li et al., 2005; Del Barrio et al., 2007; Peng et al., 2007). Analysis of the progeny of the $\mathrm{Nkx} 2.2^{+}$lineage failed to establish a lineage relationship between the p3 domain and Sox ${ }^{+}$INs (R. Taveira-Marques, N.K., and W.D.R., data not shown). Furthermore the Sox $1^{+}$

INs were generated in the appropriate number and position in embryos lacking Nkx2.2 (data not shown).

To analyze the Foxn $4^{+}$lineage, we first compared the domain of mRNA expression of the iCre transgene and the endogenous gene in adjacent E10.5 spinal cord sections and found it to be identical (supplemental Fig. 2A,B, available at www.jneurosci. org as supplemental material). Analysis of Foxn $4^{-i C r e} \times$ Rosa26 $6^{\text {stopYFP }}$ E10.5 embryonic cords showed that the ventral limit of yellow fluorescent protein (YFP) expression abuts the Olig2-expressing domain (pMN), whereas the dorsal limit is coincident with that of Nkx6.1, which defines the dorsal limit of the p2 domain (Briscoe et al., 2000) (supplemental Fig. 2C,D, available at www.jneurosci.org as supplemental material). We also confirmed that, after the specification of V2 INs, YFP expression labeled subsets of V2a and V2b INs but not other INs such as V1, suggesting that the Foxn $4^{-i C r e}$ transgene is activated in common progenitors of both V2 IN subtypes (supplemental Fig. 2E-L, available at www.jneurosci.org as supplemental material) (data not shown). A percentage of $\mathrm{Gata}^{+}$or $\mathrm{Chx} 10^{+}$cells did not express YFP (supplemental Fig. 2M-O, available at www. jneurosci.org as supplemental material), perhaps reflecting the finite time required for recombination to occur after activation of the Cre transgene, a frequent phenomenon with such iCreexpressing lines (N.K. and W.D.R., unpublished observations). To determine whether the Sox $1^{+}$INs derive from the Foxn4 lineage, we analyzed the expression of YFP relative to Sox1 in Foxn $4^{-i \text { Cre }} \times$ Rosa26 $^{\text {stopYFP }}$ embryos at E11.5 and E12.5. We found that, at both stages, some Sox ${ }^{+}$INs originated from the Foxn4 lineage (Fig. 2A,B). Cell counts revealed that the cells that coexpressed YFP (R26-YFP) and SOX1 were in the order of $30 \%$ (Fig. $2 C$ ). These data demonstrate that the Foxn $4^{+}$lineage gives rise not only to $\mathrm{V} 2 \mathrm{a} / \mathrm{b}$ INs but also to a third type of Sox1expressing INs that are quite distinct from V2a/V2b INs. We thus name these cells V2c INs.

\section{PAX6 is expressed in subsets of late-born V2 INs}

During the course of our studies, we noted that at E12.5, Pax6, in addition to its VZ expression, is expressed in neurons that migrated in the vSC. These cells are divided into two subgroups based on their migratory position. One group migrated laterally whereas another group more ventrally. Some cells in the former group expressed Chx10, whereas others expressed Gata3 (supplemental Fig. $3 A, B$, panels $a 1, b 1$, available at www.jneurosci.org as supplemental material). None of the ventrally migrating Pax6 ${ }^{+}$ cells expressed either Gata3 or Chx10 (supplemental Fig. $3 A, B$, panels a2, b2, available at www.jneurosci.org as supplemental material). Because of the proximity of these Pax $6^{+}$cells to V2c INs, we reason that these cells may also represent V2 INs. Analysis of the expression of Pax6 relative to YFP in cords derived from E12.5 and E13.5 Foxn4 ${ }^{-i \text { Cre }} \times$ Rosa2 $6^{\text {stopYFP }}$ embryos provided direct evidence that at least some of the ventral Pax6 ${ }^{+}$cells also derive from the p2 domain (supplemental Fig. 3C,D, available at www.jneurosci.org as supplemental material). However, despite their origin from the $\mathrm{p} 2$ domain, these ventral Pax $6^{+}$cells migrated to a similar position with, but were distinct from, V2c INs (supplemental Fig. $1 D$, available at www.jneurosci.org as supplemental material).

These data, along with those in the previous section, suggest that during the early stages of neurogenesis (E10.5-E12.0), the p2 domain generates at least three different types of INs distinguished by the expression of Chx10 (V2a), Gata3 (V2b), and Sox1 (V2c). At a later stage, late-born V2a and V2b INs become heterogeneous and some begin to express Pax6. In addition, some $\mathrm{Pax}^{+}$neurons also originate from the $\mathrm{p} 2$ domain and do not express Chx10, Gata3, or Sox1. Although additional genetic tools are needed to fully characterize the Pax $6^{+}$cells, our data suggest that IN diversity in the $\mathrm{p} 2$ domain is more complex than previously appreciated and point to the existence of additional IN subtypes generated from $\mathrm{p} 2$ progenitors.

\section{Sox1 is necessary to maintain the identity of V2c INs}

To examine the role of Sox 1 in the specification of V2c Ins, we compared GFP expression in heterozygote Sox $1^{\text {GFP/+ }}$ and homozygous null Sox $1^{\text {GFP/GFP }}$ embryos relative to other V2-specific markers. The number of $\mathrm{GFP}^{+}$INs in Sox ${ }^{\text {GFP/GFP }}$ cords was significantly lower than that observed in Sox ${ }^{\text {GFP/+ }}$ [phenotypically wild-type (WT)] embryos (Fig. 3A). This reduction was not associated with a similar reduction in the number of V2a or V2b INs (Fig. 3B). Despite this reduction, prospective V2c INs in Sox $1^{\text {GFP/GFP }}$ cords migrated out of the VZ but settled in a more dorsal position closer to the $\mathrm{p} 2$ domain than the $\mathrm{p} 3$ domain. Importantly, these mutant cells, unlike their heterozygous coun- 
A

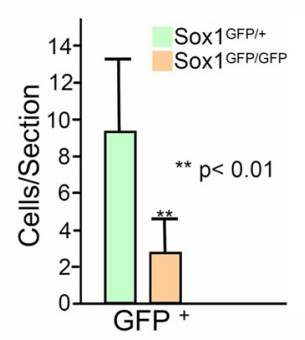

B
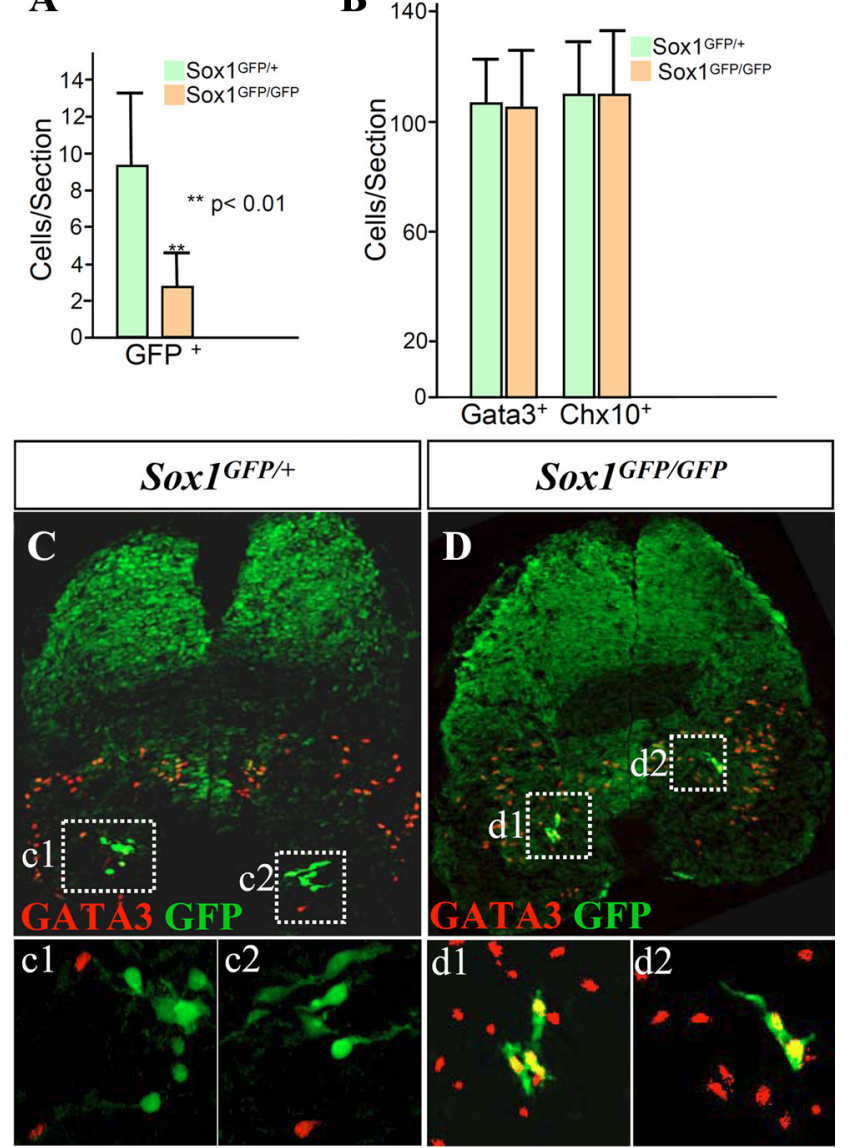

\section{$\mathbf{E}$}
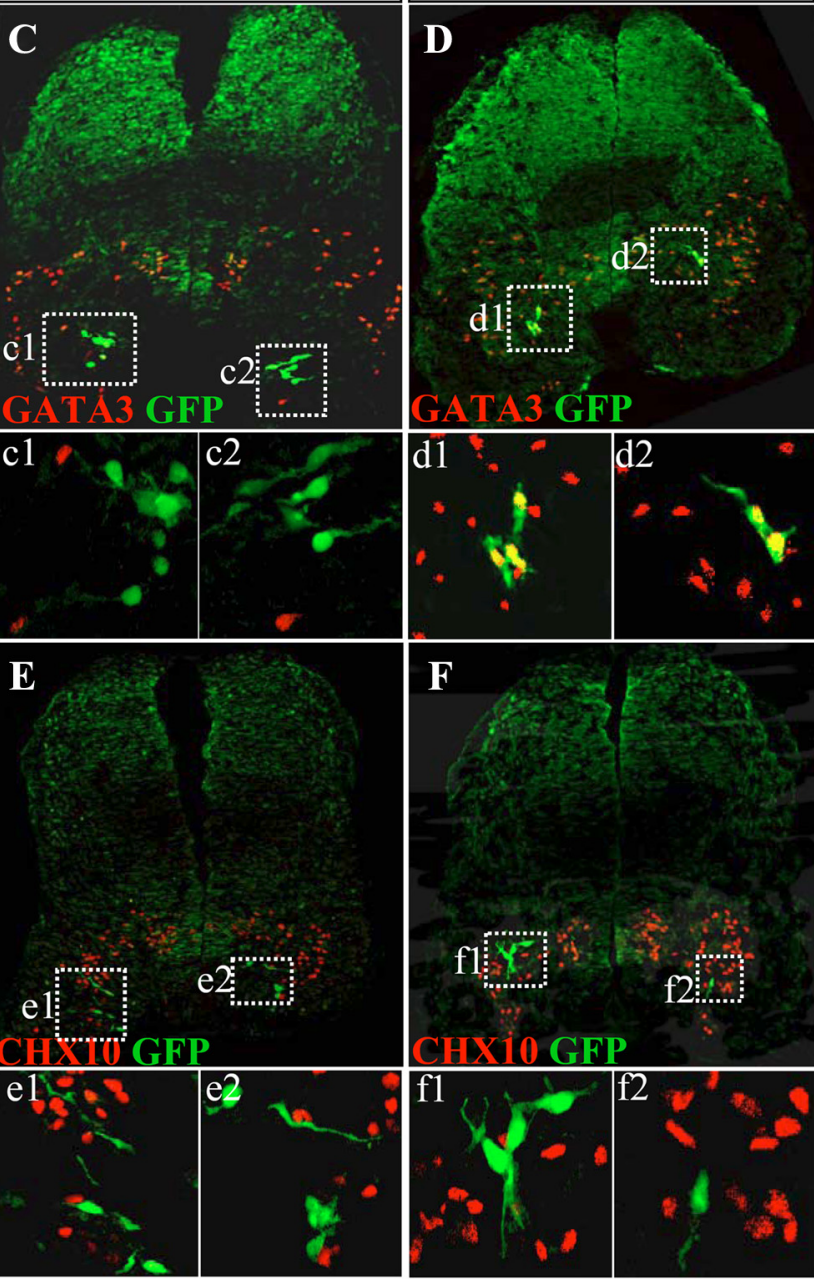

Figure 3. SOX1 determines the number and fate of V2c interneurons. $A$, Quantification of the number of V2c INs in Sox $1^{\text {GFP/+ }}$ (same as wild type) and Sox $1^{\text {GFP/GFP }}$ embryos at E12.5. Note the significant reduction of $\mathrm{GFP}^{+}$INs in mutant cords. $\boldsymbol{B}$, The reduction in the number of V2c INs was not associated with a similar reduction in Gata3 ${ }^{+}$and Chx $10^{+}$INs. C-F, Expression of GFP (V2c INs) relative to Gata3 (V2b INs) and Chx10 (V2a INs) in sections from Sox $1^{\text {GFP/+ }}$ and Sox $1^{\text {GFP/GFP }}$ embryos at E12.5. Note that, in mutant cords, GFP ${ }^{+}$neurons migrate ventrolaterally and coexpress Gata3 but not $C\left(x 10\right.$, whereas in heterozygote cords, the GFP ${ }^{+}$neurons migrate ventrally and do not express either Gata3 or Chx10. $\mathbf{1}, \mathbf{c 2}, \mathbf{d 1} \mathbf{1}, \mathbf{d 2}, \mathbf{e 1}, \mathbf{e 2}, \mathbf{f 1}$, and $\mathbf{f 2}$ are higher-magnification images of the dotted areas in $\boldsymbol{C}-\boldsymbol{F}$.

terparts, consistently expressed Gata3, suggesting that they acquired the V2b IN fate (Fig. $3 C-F$ ). Double dose of GFP in the mutant cells could not account for this observation because the same outcome was observed by comparing Sox $1^{\text {GFP/+ }}$ and Sox $1^{\text {GFP/B-geo }}$ cords at E12.5 (supplemental Fig. 4, available at www.jneurosci.org as supplemental material). These data suggest that Sox1 is specifically required for the production of the correct number of V2c INs. More importantly, Soxl is essential for the specification of the V2c IN fate in that loss of Sox 1 converts prospective V2c INs to V2b INs.

\section{V2c INs are specified in postmitotic progenitors}

Previous studies have established that IN diversity in the p2 domain is achieved in postmitotic progenitors (Li et al., 2005; Del Barrio et al., 2007; Peng et al., 2007; Joshi et al., 2009). To test whether the V2c IN fate is also attained in postmitotic progenitors, we analyzed V2c IN fate in Pax $6^{\text {Sey/Sey }}$ in which Sox1 expression is lost only in $\mathrm{VZ}$ progenitors but not in postmitotic progenitors (Genethliou et al., 2009). To achieve this, we monitored GFP expression in Pax6 $6^{\mathrm{Wt} / W t} / \mathrm{Sox} 1^{\mathrm{GFP} /+}$ and Pax6 $6^{\mathrm{Sey} / \mathrm{Sey} /}$ Sox $1^{G F P /+}$ embryos. Consistent with previous studies, we found that the number of V2a, V2b, and V2c INs was significantly reduced, most likely because of the requirement of Pax6 for generic aspects of neurogenesis in the p2 domain (Ericson et al., 1997b; Scardigli et al., 2001) (supplemental Fig. 5B, available at www. jneurosci.org as supplemental material). Importantly, although loss of Pax6 function led to complete loss of Sox1 expression in the ventral VZ, those V2c INs that were specified maintained their correct molecular identity and did not convert to Gata3-

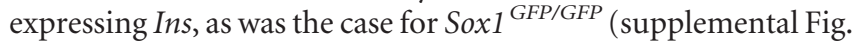
$5 F$, available at www.jneurosci.org as supplemental material). These data, combined, with those in the previous section, suggest that the function of Sox1 in specifying the V2c IN fate is important in postmitotic cells and not in VZ progenitors.

\section{V2c interneurons derive from Gata ${ }^{+}$precursors}

The analysis of the fate of V2c INs in Sox $1^{\text {GFP/GFP }}$ embryos suggested that V2c INs might be specified as Gata3 ${ }^{+}$INs because in the absence of Sox1, prospective V2c INs switch to expressing Gata3. To directly test this hypothesis, we generated stable BAC transgenic mice that express eGFP driven by Gata3 promoter/ enhancer sequences, denoted Gata3 ${ }^{-e G F P}$. Analysis of cords derived from Gata3 ${ }^{-e G F P}$ embryos at E10.5-E12.5 with several markers confirmed that eGFP expression was restricted to the V2b lineage (supplemental Fig. 6, available at www.jneurosci.org as supplemental material). eGFP is a very stable protein and can be detected in cells $24-48 \mathrm{~h}$ after gene transcription stops, depending on copy number and promoter strength. Thus, by analyzing Gata3 ${ }^{-e G F P}$ spinal cords, we could trace, over a short developmental window, any progeny of the Gata3 lineage. Because V2c INs are generated between E10.5 and E11.5 (Fig. 1E), we tested the expression of Sox1 relative to eGFP at E12.5 and E14.5 in Gata3 ${ }^{-e G F P}$ spinal cord sections. At E12.5, all Sox $1^{+}$cells outside the VZ retained GFP expression (Fig. $4 A-C$ ). However, at E14.5, long after V2c INs are specified, Sox1 and eGFP expression were mutually exclusive (Fig. $4 D-F$ ). These data suggest that V2C INs are generated from $\mathrm{Gata}^{+}{ }^{+}$-expressing cells, which subsequently loose Gata3 expression and switch on Sox1, thereby acquiring the $\mathrm{V} 2 \mathrm{c}$ IN fate.

\section{Foxn 4 is required for $\mathrm{V} 2 \mathrm{c}$ interneuron specification}

Our lineage analysis of the progeny of Foxn $4^{+}$progenitors could not establish unequivocally whether all Sox $1^{+}$INs originated from the p 2 domain because only $\sim 30 \%$ of these cells expressed YFP in Foxn $4^{-i C r e E R T 2} \times$ Rosa2 $6^{\text {stop YFP }}$ mice. Although this was most likely the result of delayed Cre-mediated recombination, we searched for additional evidence to support the hypothesis of a genetic link between the production of V2b and V2c INs. To this 
end, we analyzed the fate of Sox ${ }^{+}$INs in embryos lacking Foxn4 in which all Gata $^{+}$INs are missing and V2a INs are overproduced, most likely through a V2bto-V2a fate change (Li et al., 2005).

Between E10.5 and E12.5 in wild-type embryos, many Foxn $4^{+}$progenitors close to the ventricular zone express Sox1, and putative Sox ${ }^{+} \mathrm{V} 2 \mathrm{c}$ INs were generated in the appropriate number and position (Fig. 5A-F and data not shown). However, in Foxn4 mutant embryos at E12.5, Sox ${ }^{+}$V2c INs were completely absent at all axial levels studied (Fig. 5G-I). These data demonstrate that Sox ${ }^{+} \mathrm{V} 2 \mathrm{c}$ INs are indeed generated from Foxn4-expressing precursors. In addition, the absence of Sox ${ }^{+}$V2c INs and Gata3 ${ }^{+}$V2b but not $\mathrm{Chx}_{10}{ }^{+} \mathrm{V} 2 \mathrm{a}$ INs (Li et al., 2005) reinforces the view that V2c INs are generated from V2b INs.

\section{Discussion}

During spinal cord development, progenitors in the $\mathrm{p} 2$ domain were thought to generate only two types of INs, V2a and V2b. We show here that the transcription factor Sox 1 is required, in a cell-autonomous manner, for development of a third type of p2-derived INs, which we have named V2c.

\section{Lineage development of V2 INs}

We have shown previously that Foxn4 functions upstream of Notch signaling in all committed p 2 progenitors in which it is necessary and sufficient to activate the expression of Mash1 and Dll4 (Li et al., 2005; Del Barrio et al., 2007; Peng et al., 2007). Despite the fact that Foxn 4 is expressed in all postmitotic $\mathrm{p} 2$ progenitors, loss of this gene only affects V2b IN development, causing V2b INs to switch fate and become V2a INs (Li et al., 2005). Therefore, past studies could not provide direct evidence of the lineage relationship between the Foxn4 lineage and the production of V2 INs. In this study, we used genetic fate mapping to provide direct evidence that Foxn $4^{+}$progenitors give rise to all V2 INs identified to date.

Although Foxn4 is expressed in all V2 progenitors, we show that IN diversity is further elaborated after V2a and V2b fates are specified. We specifically show that a small subset of $\mathrm{V} 2 \mathrm{~b}$ INs gives rise to $\mathrm{V} 2 \mathrm{c}$ INs, which are indentified by expression of Sox1. The lineage relationship between V2b and $\mathrm{V} 2 \mathrm{c}$ INs is supported by the following observations. First, in Foxn4 mutant embryos, which fail to develop any V2b INs (Li et al., 2005), also fail to generate any V2c INs (this study). Second, our short-term fate map-
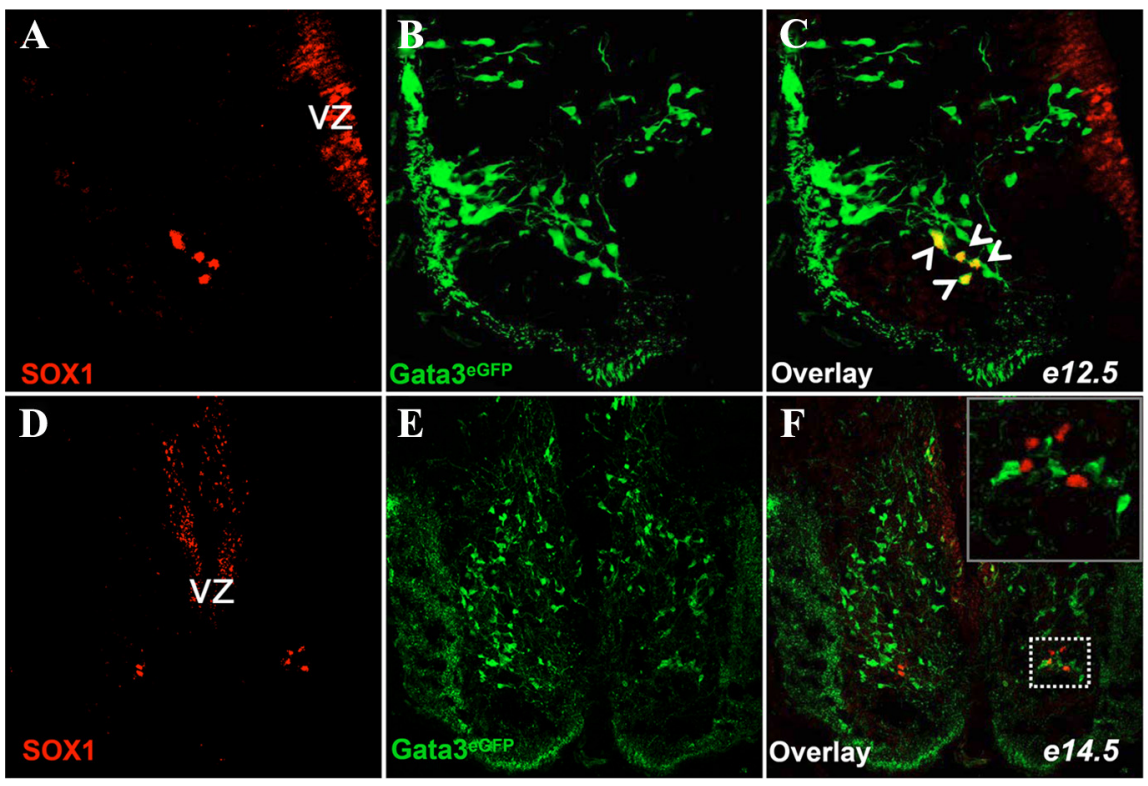

Figure 4. SOX $1^{+}$INs derive from Gata3 ${ }^{+}$progenitors. Spinal cord sections from Gata ${ }^{\text {-eGFP }}$ embryonic spinal cords at E12.5 $(\boldsymbol{A}-\boldsymbol{C})$ and E14.5 (D-F) stained for anti-GFP and anti-Sox1 antibodies. In this experiment, eGFP is used as a short-term marker for tracing derivative cell populations from the Gata ${ }^{+}{ }^{+}$lineage. Note that, at E12.5, all Sox ${ }^{+}{ }^{+}$cells are also GFP ${ }^{+}$, whereas at E14.5, no double-positive cells are observed.
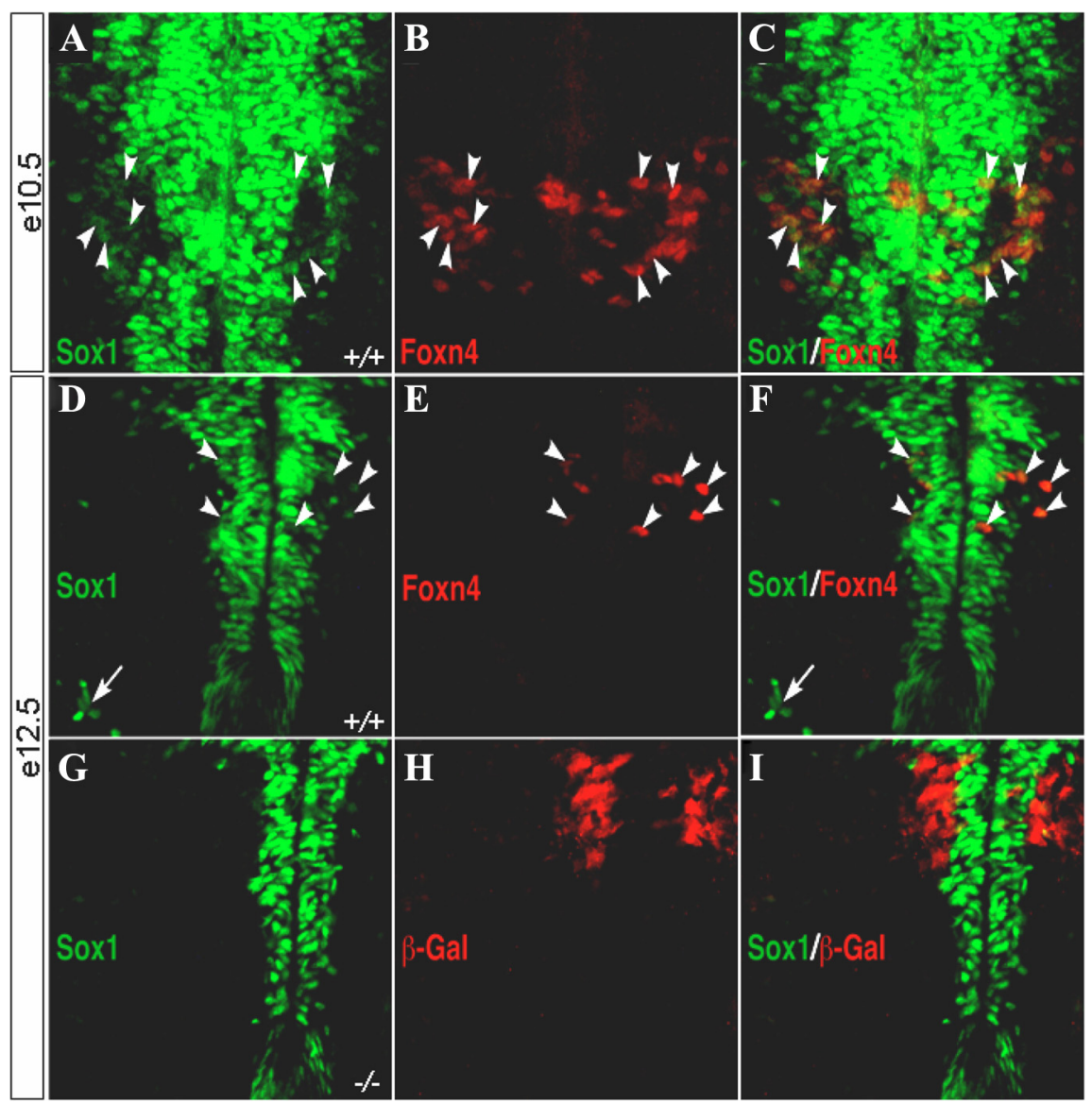

Figure 5. The production of V2c INs depends on Foxn4. Spinal cord sections from E10.5 $(\boldsymbol{A}-\boldsymbol{C})$ and E12.5 $(\boldsymbol{D}, \boldsymbol{E})$ wild-type embryos and E12.5 mutant embryos $(\mathbf{G}-\boldsymbol{I})$ were immunolabeled with the indicated antibodies. All panels show sections of the vSC. A-F represent wild-type (Foxn4 ${ }^{+/+}$) embryos, and $\mathbf{G}-\mathbf{I}$ represent mutant (Foxn4 ${ }^{\text {lacz/lacz }}$ ) embryos. In the latter, $\beta$-galactosidase ( $\beta$-Gal) defines Foxn4-expressing cells (Li et al., 2004). Note that, in E12.5 Foxn4 ${ }^{+/+}$, the Sox1-immunoreactive V2c INs (arrows in $\boldsymbol{D}$ and $\boldsymbol{F}$ ) are clustered bilaterally in the ventral cord but are completely missing from Foxn $4^{\text {lacz/lacz }}$ cords $(\mathbf{G}-\boldsymbol{I})$. Arrowheads indicate coexpressing cells. 


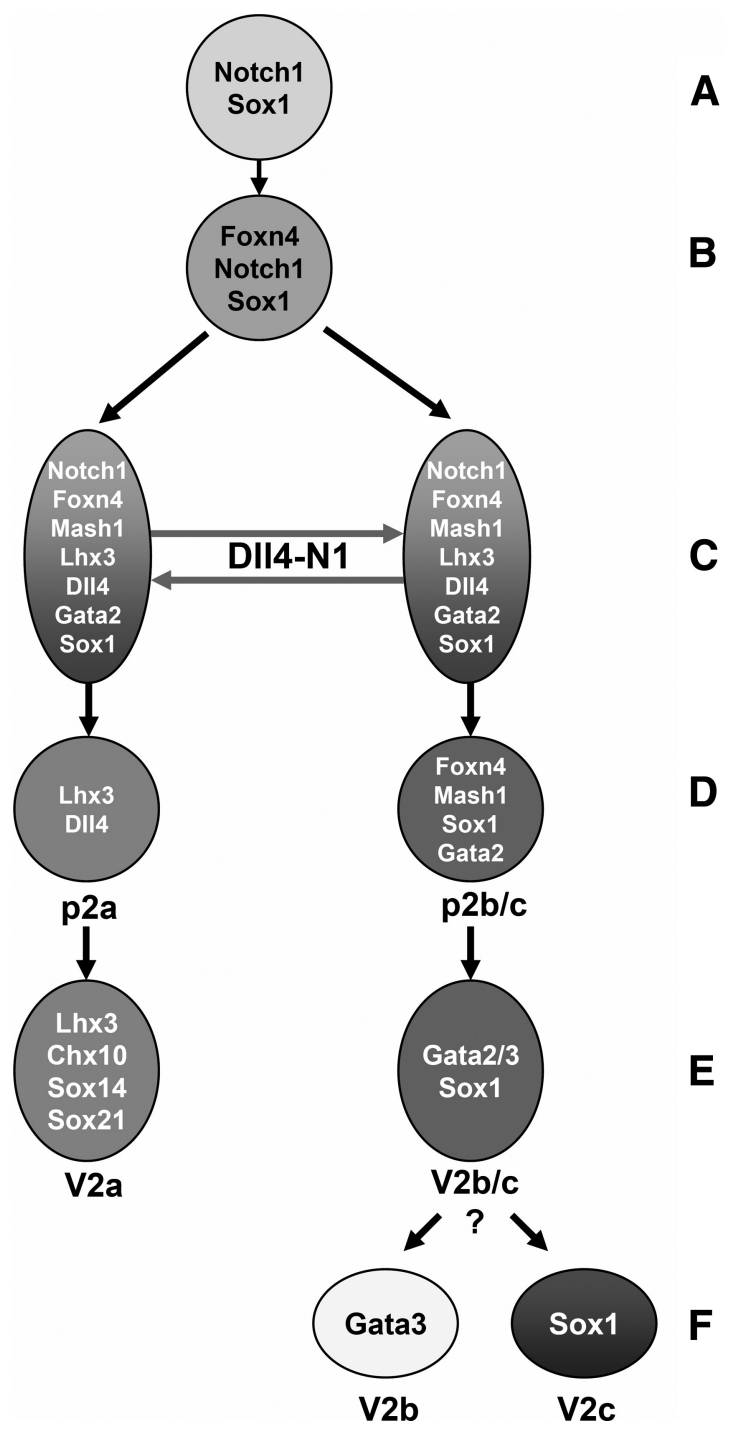

Figure 6. Model for V2 INs specification. This model represents a refinement of a previous model (Del Barrio et al., 2007). All p2 neuroepithelial progenitors express Sox 1 and Notch-1 ( $\boldsymbol{A})$. Some of these switch on Foxn4 and continue to express Sox1/Notch-1 $(\boldsymbol{B})$. Foxn4 induces the expression of DII4/Mash1/Gata2, whereas Lhx 3 is also switched on (C). Reciprocal interactions between DII/Notch1 produce two populations of progenitors (D): p2a progenitors expressing D114/Lhx3 and p2b/c progenitors expressing Foxn4/Mash1/Gata2/Sox1 (and activated Notch). p2a/b progenitors switch on Gata3 and subsequently segregate into V2b and V2c INs. The mechanism that segregates V2b and V2c IN fates is unknown (denoted by a question mark).

ping using Gata3 ${ }^{-G F P}$ embryos revealed that V2c INs transiently express Gata3, which is then switched off as the cells migrate in the gray matter. Third, loss of Sox 1 causes prospective V2c INs to maintain Gata3 expression and acquire a V2b IN fate.

Previous studies have shown that the mechanism that allocates the V2a versus V2b IN fate is mediated by Dll4/Notch1 interactions in Foxn4-expressing neuronal progenitors (Del Barrio et al., 2007; Peng et al., 2007). After this binary fate choice, the action of the Lim-only protein (Lmo4) in subsets of postmitotic progenitors either favors or inhibits transcriptional complexes and consolidates the V2a and V2b IN fates (Joshi et al., 2009). Our data support a model whereby an additional mechanism exists that diversifies further V2b INs and generates at least one sublineage, the V2c INs (Fig. 6). By analyzing Pax6-mutant embryos that lack Sox1 expression in the VZ, we show that V2c INs are still generated. Hence, the mechanism that determines the fate of V2c INs is directly linked to the regulation of Sox1 in postmitotic progenitors and must be distinct from the regulation of Sox1 in uncommitted progenitors (Fig. 6). The molecular components of this mechanism are presently unknown.

\section{Acquisition of V2 interneuron subtype identity}

Several studies have provided strong evidence that the acquisition of V2 IN subtype identity is determined in postmitotic V2 progenitors. For instance, loss of Foxn4 leads to a V2b $\rightarrow$ V2a IN fate switch caused by loss of Dll4 expression, resulting in persistent Notch expression in all p2 progenitors (Li et al., 2005; Del Barrio et al., 2007; Peng et al., 2007). Likewise, loss of Lmo4 and hemizygosity for stem cell leukemia factor $(S c l)$ (a marker for V2b progenitors) also leads to overproduction of V2a INs at the expense of V2b INs (Joshi et al., 2009). Our data further support the hypothesis that V2 IN diversification takes place in postmitotic progenitors and that V2 INs remain plastic for some time after adopting cell identities via Delta/Notch signaling. These observations suggest that additional transcription factors expressed in postmitotic V2 cells are required to consolidate the V2 IN subtype identity, most likely by regulating Lmo4 expression in progenitors of V2 INs (Joshi et al., 2009). SoxB factors might play a role in this process. Sox 14 and Sox 21 belong to Group B of the Sox gene family and function as transcriptional repressors, unlike Sox1, which functions as an activator of seemingly the same set of target genes (Sandberg et al., 2005). Sox14 and Sox21 are both expressed in V2a INs (Hargrave et al., 2000; Sandberg et al., 2005) in which they appear to have a redundant function (S.M., unpublished data). This observation raises the possibility that SoxB factors with opposing transcriptional activities expressed in distinct p2-derived IN subtypes may function to consolidate alternative V2 IN fates by activating or repressing of SoxB target genes in postmitotic progenitors.

\section{Multiple functions of Pax6 in the vSC}

In addition to its role in patterning (Briscoe et al., 2000) and neuronal specification (Scardigli et al., 2001), Pax6 specifically regulates Sox1 expression in neuroepithelial progenitors in the vSC but not in V2c INs (Genethliou et al., 2009; this study). Recently, Pax6 has been shown to regulate neurogenesis at two distinct stages in mice and chick (Bel-Vialar et al., 2007). In the first stage, it induces neurogenesis by promoting Neurogenin 2 (Ngn2) expression, and, in a second step, it blocks neurogenesis downstream of Ngn2, a function also ascribed to SoxB1 genes (Bylund et al., 2003; Sandberg et al., 2005). It is therefore possible that, in some progenitor domains, a Pax6 $\rightarrow$ Sox 1 cascade exists as part of a mechanism to block neuronal commitment per se. This mechanism must be both stage and context specific because, during neurogenesis in the p2 domain, the expression of Sox 1 and Pax6 in postmitotic progenitors segregate.

Although the vast majority of V2 INs are generated between E10.5 and E12.5, some V2a and V2b INs that emerge from the VZ at E12.5 express Pax6 (supplemental Fig. 3, available at www. jneurosci.org as supplemental material). These observations suggest that late-born V2a and V2b INs are heterogeneous. The identification of more molecular markers for early-born V2 INs should shed light if these cells are also heterogeneous, raising the question of how such heterogeneity could be controlled at the molecular level in light of a recently proposed model for segregating V2 IN fates (Joshi et al., 2009). In addition to the expression of Pax6 in subsets of V2 INs, some Pax6 ${ }^{+}$neurons migrate ventrally close to V2c INs and do not express any known V2specific marker. At least some of these cells still derive from the $\mathrm{p} 2$ 
domain. We are currently investigating further the origin and fate of these Pax $6^{+}$postmitotic cells using additional genetic tools.

It is nevertheless intriguing that, during the migration of $\mathrm{Pax}^{+}$neurons in the vSC, the p2 domain begins to generate astrocyte progenitors (Pringle et al., 2003; Hochstim et al., 2008; Genethliou et al., 2009). This observation suggests that there must be a mechanism to control the simultaneous allocation of neuronal and astrocytic fates from $\mathrm{p} 2$ progenitors. It has been proposed that the transcription factor SCL regulates, cell autonomously, both the production of V2b INs and astrocytes in the p2 domain (Muroyama et al., 2005). Although the production of V2b INs and the expression of SCL depend on Foxn4 (Del Barrio et al., 2007), our data suggest that the Foxn4 lineage gives rises only to V2b INs but not astrocytes (S.M., unpublished data). Also the production of Pax6 ${ }^{+}$neurons does not require Foxn4 function (M.X., unpublished data). Thus, it is unlikely that the production of V2b INs is lineally related to the production of astrocytes. It is more likely that neurogenesis and astrogenesis in the $\mathrm{p} 2$ domain are regulated by mechanisms that operate independent of Foxn 4 and the function of $\mathrm{Scl}$ on astrocyte specification may not be cell autonomous.

\section{References}

Aubert J, Stavridis MP, Tweedie S, O'Reilly M, Vierlinger K, Li M, Ghazal P, Pratt T, Mason JO, Roy D, Smith A (2003) Screening for mammalian neural genes via fluorescence-activated cell sorter purification of neural precursors from Soxl-gfp knock-in mice. Proc Natl Acad Sci U S A 100 [Suppl 1]:11836-11841.

Batista MF, Jacobstein J, Lewis KE (2008) Zebrafish V2 cells develop into excitatory CiD and Notch signalling dependent inhibitory VeLD interneurons. Dev Biol 322:263-275.

Bel-Vialar S, Medevielle F, Pituello F (2007) The on/off of Pax6 controls the tempo of neuronal differentiation in the developing spinal cord. Dev Biol 305:659-673.

Briscoe J, Sussel L, Serup P, Hartigan-O’Connor D, Jessell TM, Rubenstein JL, Ericson J (1999) Homeobox gene Nkx2.2 and specification of neuronal identity by graded Sonic hedgehog signalling. Nature 398:622-627.

Briscoe J, Pierani A, Jessell TM, Ericson J (2000) A homeodomain protein code specifies progenitor cell identity and neuronal fate in the ventral neural tube. Cell 101:435-445.

Bylund M, Andersson E, Novitch BG, Muhr J (2003) Vertebrate neurogenesis is counteracted by Sox1-3 activity. Nat Neurosci 6:1162-1168.

Dalla Torre di Sanguinetto SA, Dasen JS, Arber S (2008) Transcriptional mechanisms controlling motor neuron diversity and connectivity. Curr Opin Neurobiol 18:36-43.

Del Barrio MG, Taveira-Marques R, Muroyama Y, Yuk DI, Li S, WinesSamuelson M, Shen J, Smith HK, Xiang M, Rowitch D, Richardson WD (2007) A regulatory network involving Foxn4, Mash1 and delta-like 4/Notch1 generates V2a and V2b spinal interneurons from a common progenitor pool. Development 134:3427-3436.

Ekonomou A, Kazanis I, Malas S, Wood H, Alifragis P, Denaxa M, Karagogeos D, Constanti A, Lovell-Badge R, Episkopou V (2005) Neuronal migration and ventral subtype identity in the telencephalon depend on SOX1. PLoS Biol 3:e186.

Ericson J, Briscoe J, Rashbass P, van Heyningen V, Jessell TM (1997a) Graded sonic hedgehog signaling and the specification of cell fate in the ventral neural tube. Cold Spring Harb Symp Quant Biol 62:451-466.

Ericson J, Rashbass P, Schedl A, Brenner-Morton S, Kawakami A, van Heyningen V, Jessell TM, Briscoe J (1997b) Pax6 controls progenitor cell identity and neuronal fate in response to graded Shh signaling. Cell 90:169-180.

Genethliou N, Panayiotou E, Panayi H, Orford M, Mean R, Lapathitis G, Gill H, Raoof S, De Gasperi R, Elder G, Kessaris N, Richardson WD, Malas S (2009) SOX1 links the function of neural patterning and Notch signalling in the ventral spinal cord during the neuron-glial fate switch. Biochem Biophys Res Commun 390:1114-1120.

Hargrave M, Karunaratne A, Cox L, Wood S, Koopman P, Yamada T (2000) The HMG box transcription factor gene Sox14 marks a novel subset of ventral interneurons and is regulated by sonic hedgehog. Dev Biol 219:142-153.
Hill RE, Favor J, Hogan BL, Ton CC, Saunders GF, Hanson IM, Prosser J, Jordan T, Hastie ND, van Heyningen V (1991) Mouse small eye results from mutations in a paired-like homeobox-containing gene. Nature 354:522-525.

Hochstim C, Deneen B, Lukaszewicz A, Zhou Q, Anderson DJ (2008) Identification of positionally distinct astrocyte subtypes whose identities are specified by a homeodomain code. Cell 133:510-522.

Joshi K, Lee S, Lee B, Lee JW, Lee SK (2009) LMO4 controls the balance between excitatory and inhibitory spinal V2 interneurons. Neuron 61:839-851.

Karunaratne A, Hargrave M, Poh A, Yamada T (2002) GATA proteins identify a novel ventral interneuron subclass in the developing chick spinal cord. Dev Biol 249:30-43.

Kimura Y, Satou C, Higashijima S (2008) V2a and V2b neurons are generated by the final divisions of pair-producing progenitors in the zebrafish spinal cord. Development 135:3001-3005.

Li S, Mo Z, Yang X, Price SM, Shen MM, Xiang M (2004) Foxn4 controls the genesis of amacrine and horizontal cells by retinal progenitors. Neuron 43:795-807.

Li S, Misra K, Matise MP, Xiang M (2005) Foxn4 acts synergistically with Mash1 to specify subtype identity of V2 interneurons in the spinal cord. Proc Natl Acad Sci U S A 102:10688-10693.

Lundfald L, Restrepo CE, Butt SJ, Peng CY, Droho S, Endo T, Zeilhofer HU, Sharma K, Kiehn O (2007) Phenotype of V2-derived interneurons and their relationship to the axon guidance molecule EphA4 in the developing mouse spinal cord. Eur J Neurosci 26:2989-3002.

Malas S, Postlethwaite M, Ekonomou A, Whalley B, Nishiguchi S, Wood H, Meldrum B, Constanti A, Episkopou V (2003) Sox1-deficient mice suffer from epilepsy associated with abnormal ventral forebrain development and olfactory cortex hyperexcitability. Neuroscience 119:421-432.

Masahira N, Takebayashi H, Ono K, Watanabe K, Ding L, Furusho M, Ogawa Y, Nabeshima Y, Alvarez-Buylla A, Shimizu K, Ikenaka K (2006) Olig2positive progenitors in the embryonic spinal cord give rise not only to motoneurons and oligodendrocytes, but also to a subset of astrocytes and ependymal cells. Dev Biol 293:358-369.

Moran-Rivard L, Kagawa T, Saueressig H, Gross MK, Burrill J, Goulding M (2001) Evx1 is a postmitotic determinant of v0 interneuron identity in the spinal cord. Neuron 29:385-399.

Muhr J, Andersson E, Persson M, Jessell TM, Ericson J (2001) Grouchomediated transcriptional repression establishes progenitor cell pattern and neuronal fate in the ventral neural tube. Cell 104:861-873.

Muroyama Y, Fujiwara Y, Orkin SH, Rowitch DH (2005) Specification of astrocytes by bHLH protein SCL in a restricted region of the neural tube. Nature 438:360-363.

Peng CY, Yajima H, Burns CE, Zon LI, Sisodia SS, Pfaff SL, Sharma K (2007) Notch and MAML signaling drives Scl-dependent interneuron diversity in the spinal cord. Neuron 53:813-827.

Pevny LH, Sockanathan S, Placzek M, Lovell-Badge R (1998) A role for SOX1 in neural determination. Development 125:1967-1978.

Pringle NP, Yu WP, Howell M, Colvin JS, Ornitz DM, Richardson WD (2003) Fgfr3 expression by astrocytes and their precursors: evidence that astrocytes and oligodendrocytes originate in distinct neuroepithelial domains. Development 130:93-102.

Sandberg M, Källström M, Muhr J (2005) Sox21 promotes the progression of vertebrate neurogenesis. Nat Neurosci 8:995-1001.

Scardigli R, Schuurmans C, Gradwohl G, Guillemot F (2001) Crossregulation between Neurogenin 2 and pathways specifying neuronal identity in the spinal cord. Neuron 31:203-217.

Srinivas S, Watanabe T, Lin CS, William CM, Tanabe Y, Jessell TM, Costantini F (2001) Cre reporter strains produced by targeted insertion of EYFP and ECFP into the ROSA26 locus. BMC Dev Biol 1:4.

Thaler JP, Lee SK, Jurata LW, Gill GN, Pfaff SL (2002) LIM factor Lhx3 contributes to the specification of motor neuron and interneuron identity through cell-type-specific protein-protein interactions. Cell 110:237-249.

Wood HB, Episkopou V (1999) Comparative expression of the mouse Sox1, Sox 2 and Sox3 genes from pre-gastrulation to early somite stages. Mech Dev 86:197-201.

Yang X, Tomita T, Wines-Samuelson M, Beglopoulos V, Tansey MG, Kopan $\mathrm{R}$, Shen J (2006) Notch1 signaling influences v2 interneuron and motor neuron development in the spinal cord. Dev Neurosci 28:102-117.

Zhang Y, Muyrers JP, Testa G, Stewart AF (2000) DNA cloning by homologous recombination in Escherichia coli. Nat Biotechnol 18:1314-1317. 